\title{
Article
}

\section{Evidence for Novel Action at the Cell- Binding Site of Human Angiogenin Revealed by Heteronuclear NMR Spectroscopy and in silico and in vivo Studies}

Chatzileontiadou, Demetra S. M., Tsika, Aikaterini C., Diamantopoulou, Zoi, Delbé, Jean, Badet, Josette, Courty, José, Skamnaki, Vassiliki T., Parmenopoulou, Vanessa, Komiotis, Dimitri, Hayes, Joseph, Spyroulias, Georgios A. and Leonidas, Demetres D.

Available at https://clok.uclan.ac.uk/21400/

Chatzileontiadou, Demetra S. M., Tsika, Aikaterini C., Diamantopoulou, Zoi, Delbé, Jean, Badet, Josette, Courty, José, Skamnaki, Vassiliki T., Parmenopoulou, Vanessa, Komiotis, Dimitri et al (2018) Evidence for Novel Action at the Cell-Binding Site of Human Angiogenin Revealed by Heteronuclear NMR Spectroscopy and in silico and in vivo Studies. ChemMedChem, 13 (3). pp. 259-269. ISSN 1860-7179

It is advisable to refer to the publisher's version if you intend to cite from the work. http://dx.doi.org/10.1002/cmdc.201700688

For more information about UCLan's research in this area go to http://www.uclan.ac.uk/researchgroups/ and search for < name of research Group>.

For information about Research generally at UCLan please go to http://www.uclan.ac.uk/research/

All outputs in CLoK are protected by Intellectual Property Rights law, including Copyright law. Copyright, IPR and Moral Rights for the works on this site are retained by the individual authors and/or other copyright owners. Terms and conditions for use of this material are defined in the policies page. 


\section{Evidence for novel action at the cell binding site of human Angiogenin revealed by heteronuclear NMR spectroscopy, in silico and in vivo studies}

Demetra S.M. Chatzileontiadou ${ }^{[a]}$, Aikaterini C. Tsika ${ }^{[b]}$, Zoi Diamantopoulou ${ }^{[c]}$, Jean Delbé[c], Josette Badet $^{[\mathrm{d}]}$, José Courty ${ }^{[c]}$, Vassiliki T. Skamnaki[a], Vanessa Parmenopoulou ${ }^{[a]}$, Dimitri Komiotis ${ }^{[a]}$, Joseph M. Hayes ${ }^{*}[e]$, Georgios A. Spyroulias ${ }^{\star}[b]$, and Demetres D. Leonidas ${ }^{*}[a]$

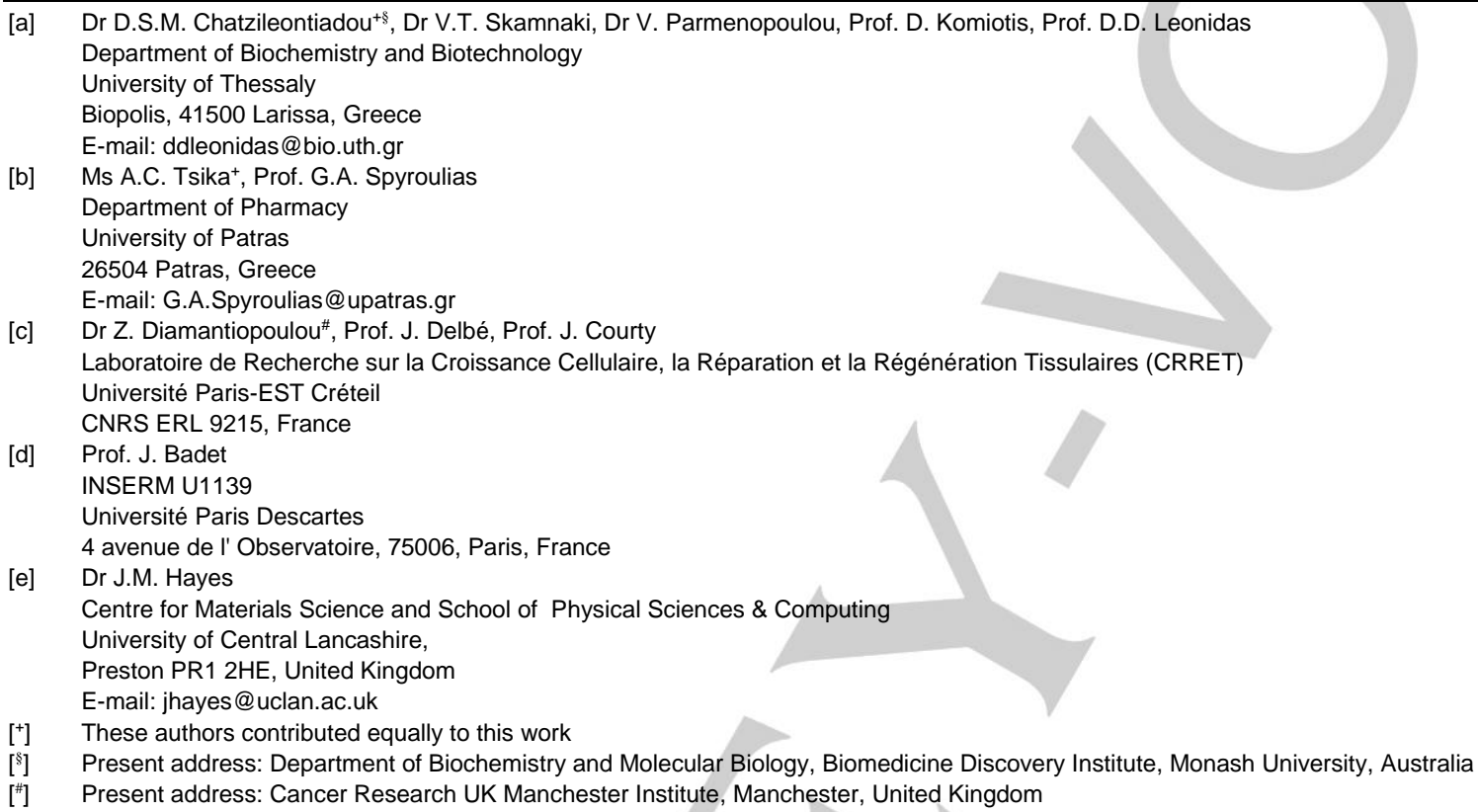

Supporting information for this article is given via a link at the end of the document.

\begin{abstract}
A member of Ribonuclease A superfamily, human Angiogenin (hAng) is a potent angiogenic factor. Heteronuclear NMR spectroscopy combined with induced-fit docking revealed a dual binding mode for the most antiangiogenic compound of a series of ribofuranosyl pyrimidine nucleosides that strongly inhibit hAng's angiogenic activity in vivo. While modelling suggests the potential of a simultaneous binding of the inhibitors at the active and cell binding sites, NMR indicates greater affinity for the cell binding site than for the active site. Additionally, a 100 nanoseconds molecular dynamics simulation have confirmed the stability of the binding at the cellbinding site with the predicted protein-ligand interactions in excellent agreement with the NMR data. This is the first time that a nucleoside inhibitor is reported to inhibit completely the angiogenic activity of hAng in vivo by exerting dual inhibitory activity on hAng, blocking both the entrance of hAng into the cell and the ribonucleolytic activity.
\end{abstract}

\section{Introduction}

Human angiogenin ( $\mathrm{hAng}$ ) is a basic protein containing 123 amino acids with a molecular weight of about $14.4 \mathrm{kDa}{ }^{[1]}$. It is the 5 th member of the pancreatic ribonuclease (RNase) superfamily having a $33 \%$ amino acid identity and $56 \%$ overall homology to that of RNase A, the prototypic member of this superfamily ${ }^{[2]}$. Angiogenin is an angiogenic factor, playing a major role in the growth and establishment of human tumors, whereas RNase A is not ${ }^{[3]}$. In the extracellular environment, secreted hAng binds to a $170-\mathrm{kDa}$ transmembrane protein that has yet to be fully characterized, activating many signal transduction pathways ${ }^{[4]}$ Extracellular hAng also interacts with cell-surface actin promoting extracellular matrix degradation allowing thus cell migration and invasion ${ }^{[4]}$. Under growth conditions hAng accumulates in the nucleus/nucleolus promoting mRNA transcription and rRNA transcription/procession ${ }^{[4-5]}$, while under stress conditions it accumulates in the cytoplasm where it forms tiRNAs leading to the inhibition of translational initiation ${ }^{[6]}$. hAng is implicated in almost every step of tumorigenesis since it promotes tumour cell survival, proliferation, adhesion, migration and invasion, while nuclear hAng is necessary for angiogenesis induced by other angiogenic factors ${ }^{[7]}$. In addition to the ribonucleolytic active site and the cell-binding site of hAng (residues 60-68 and 108-111), a third region has been implicated as necessary for angiogenic activity, the nuclear localisation signal (31RRRGL35) ${ }^{[8]}$.

Importantly, angiogenin presents a ribonucleolytic activity which is significantly lower than that of RNase A but essential for its 
angiogenic activity ${ }^{[9]}$. Therefore, hAng has been suggested as a pharmaceutical target for the development of small molecule inhibitors of its enzymatic activity as novel anti-angiogenic agents ${ }^{[10]}$. The active site of hAng contains various binding subsites for the phosphate, base, and ribose moieties of the RNA substrate (designated $P_{0} \ldots P_{n}, B_{0} \ldots B_{n}$ and $R_{0} \ldots R_{n}$, respectively) ${ }^{[8]}$. The central sites are $\mathrm{P}_{1}$, where $\mathrm{P}-\mathrm{O}^{5}$ bond cleavage occurs; $\mathrm{B}_{1}$, which binds the $3^{\prime}$ pyrimidine; and $B_{2}$, which interacts with the base of the $5^{\prime}$ nucleotide (Figure 1 ). Subsite $B_{1}$ shows selectivity for pyrimidines while $B_{2}$ prefers purines ${ }^{[8]}$.

The three catalytic residues of RNase A, His12, Lys41, and His 119 of the P1 subsite are conserved in hAng as His13, Lys40 and His $114{ }^{[8]}$. The key B1 residues, Thr45 and Phe120 in RNase A, are also maintained in hAng as Thr44 and Leu115. The B2 subsite is also partially conserved between hAng and RNase A. The residues that form the P2 subsite in hAng are His8 and Thr11 ${ }^{[11]}$, the structural analogues of Lys7 and Arg10 in RNase $\mathrm{A}^{[12]}$, while Leu115, Asp116, Gln117, and Arg121, in hAng, may constitute the subsite $\mathrm{PO}^{[11]}$ (Figure 1).

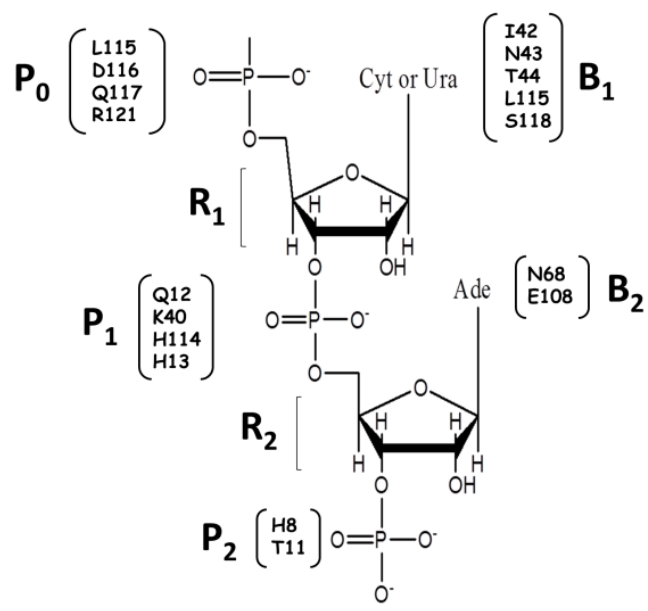

Figure 1. Schematic diagram of the subsites of the hAng active site. The residues shown were seen in various crystal and NMR structures to be within the sites $^{[8,11,13]}$

There are several three-dimensional structures of hAng, including crystal structures of the free enzyme ${ }^{[8,14]}$, crystal structures of complexes with small inorganic molecules (phosphate, pyrophosphate and sulphate ions $)^{[11,15]}$, and one solution structure by NMR spectroscopy ${ }^{[13]}$. However, all these structures display a closed conformation for the active site, where the key pyrimidine-binding subsite, $\mathrm{B}_{1}$, is obstructed by the $\mathrm{C}$-terminal segment of hAng. This blockage and the concomitant low enzymatic activity of $h A n g$, is achieved primarily by the positioning of $\mathrm{G} \ln 117$ within the $\mathrm{B}_{1}$ pyrimidine binding pocket ${ }^{[14 a]}$. Due to this fact, there is no structural information on hAng-nucleotide complexes, in contrast to other members of the RNase A superfamily. The relationship between the ribonucleolytic and angiogenic activities of hAng implies that potent and selective inhibitors of its enzymatic action will be also able to inhibit its biological activity. This has been shown so far, for a monoclonal antibody [16], an antisense oligodeoxynucleotide [17], peptides complementary to the receptor binding site of hAng ${ }^{[18]}$, and a small molecule identified by $\mathrm{HTS}$ of the $\mathrm{NCl}$ and ChemBridge libraries ${ }^{[10 c, 19]}$. The structure function relationship of $h A n g$ and the high degree of conservation between the active sites of hAng and RNase $A^{[20]}$, implies that a strategy to develop specific hAng inhibitors using as template RNase A inhibitors might be plausible. We have tested the validity of this strategy by the evaluation of a series of RNase A inhibitors (ribofuranosyl pyrimidine nucleosides and their corresponding 1,2,3-triazole derivatives) ${ }^{[21]}$ for their antiangiogenic potency in vivo. One of the most potent compounds, $\mathbf{4 a}$, and it's analogue without the triazole ring, $\mathbf{6 a}$, have been studied further for their hAng ribonucleolytic inhibitory potency in vitro. Furthermore, NMR spectroscopy using bindinginduced resonance perturbations of ${ }^{15} \mathrm{~N}$ - and ${ }^{15} \mathrm{~N} /{ }^{13} \mathrm{C}$-labeled hAng together with molecular modelling studies have been used to predict the structural mode of binding of $4 a$ to hAng. In this respect, induced-fit docking (IFD) calculations have revealed binding interactions of the compound in excellent agreement with the NMR data, including novel binding of $\mathbf{4 a}$ at the hAng 'cell binding site' confirmed by molecular dynamics (MD) simulation. This provides considerable support for targeting this binding site of hAng as a new strategy for developing better anticancer drugs.

\section{Results and Discussion}

\section{In vivo studies}

The ability of compounds $4 a-4 e$ and $6 a-6 e$ (Table 1) [21] to inhibit angiogenin induced angiogenesis using the chick embryo chorioallantoic membrane (CAM) assay [22] has been assessed using $2 \mathrm{nM}$ of Pyr1-hAng (the native form of hAng with a pyroglutamate residue at the $\mathrm{N}$-terminal) and $6 \mathrm{nM}$ of each ligand (Pyr1-hAng: ligand ratio = 1:3). The compounds did not show any effects on angiogenesis in the absence of Pyr1-hAng (data not shown). Results are presented in Figures 2 and 3 . As it is evident Pyr1-hAng displays strong angiogenic activity (Figure 2) and its presence almost doubles the number of new vessels formed (Figure 3 ) in CAM. This activity is completely inhibited by $\mathbf{4 a}, \mathbf{4 c}$, $4 e$, and $6 a$ while $4 b, 4 d, 6 b, 6 c, 6 d$, and $6 e$ partially inhibited it (Figures 2 and 3 ). The inhibitors were also tested at a concentration of $0.6 \mathrm{nM}$ (Pyr1-hAng: ligand ratio = 10:3), but no effect was observed. Therefore, it seems that the inhibitory effect is dose dependent. The in vivo inhibitory potency pattern of these compounds does not seem to follow their enzymatic inhibitory potency in RNase A [21]. However, the most potent compound, $4 \mathbf{a}$, with a Ki value of $1.6 \mu \mathrm{M}$ for RNase A (Table 1) completely inhibits the biological activity of Pyr1-hAng in the CAM assay.

The presence of the triazole group seems to influence significantly the anti-angiogenic potency. Thus, in general 1,2,3-triazole 5substituted uracil furanonucleosides $\mathbf{4 a - e}$ are more potent than the $\mathrm{C}-5$ substituted uridines $6 \mathrm{a}-\mathrm{e}$. However, C-5 substituents $\mathrm{H}$ and $\mathrm{Br}$, seem to increase the inhibitory potency regardless the existence of the triazole linker since ribofuranosyl pyrimidine nucleosides $6 \mathbf{a}$ and $6 \mathbf{c}$, and their corresponding 1,2,3-triazole derivatives, $\mathbf{4 a}$ and $\mathbf{4 c}$, bearing respectively the same group at the C-5 position inhibit Pyr1-hAng activity at the same extent. 
Table 1. The chemical structures of the inhibitors studied together with their $K$ values for RNase A [ ${ }^{21]}$

\begin{tabular}{|c|c|c|c|}
\hline & Triazole nucleoside & Nucleoside & \\
\hline & $\mathrm{OH} \quad \mathrm{OH}$ & $\prod_{\mathrm{OH}}$ & o \\
\hline $\begin{array}{l}\text { C-5-Substituent } \\
\text { X) }\end{array}$ & Compound $\quad K_{\mathrm{i}}(\mu \mathrm{M})$ & Compound & $K_{\mathrm{i}}(\mu \mathrm{M})$ \\
\hline $\mathrm{H}$ & $1.6 \pm 0.2$ & $6 a$ & $\begin{array}{ll}28.5 \quad \pm \\
4.5\end{array}$ \\
\hline $\mathrm{Me}$ & $25.8 \pm 2.4$ & $6 b$ & $\begin{array}{l}44.1 \quad \pm \\
6.4\end{array}$ \\
\hline $\mathrm{Br}$ & $\begin{array}{l}20 \% \\
\text { inhibition } \\
\text { at } 50 \mu \mathrm{M}\end{array}$ & $6 c$ & $\begin{array}{l}69.1 \pm \\
2.1\end{array}$ \\
\hline $\mathrm{Cl}$ & $94.8 \pm 8.7$ & $6 d$ & $\begin{array}{l}44.5 \quad \pm \\
4.6\end{array}$ \\
\hline $\mathrm{F}$ & $30.8 \pm 4.4$ & $6 e$ & $\begin{array}{l}33.6 \quad \pm \\
2.6\end{array}$ \\
\hline
\end{tabular}

The influence of the triazole linker is evident when examining the effect of the $\mathrm{C}-5$ substituents $(\mathrm{H}, \mathrm{Me}, \mathrm{Br}, \mathrm{Cl}$, and $\mathrm{F})$. The presence of $\mathrm{F}, \mathrm{Cl}$, or Me seems to weaken the inhibitory potency of the nucleosides (Figure 3) whereas the inclusion of the triazole linker seems to counteract this effect and $4 a, 4 c, 4 e, 6 a$ and $6 e$ display equivalent activities. Thus, the ribofuranosyl pyrimidine nucleosides having these $\mathrm{C}-5$ groups display a weaker inhibitory potency than their corresponding 1,2,3-triazole derivatives (Figures 2 and 3 ).

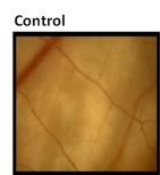

hAng $+6 b$

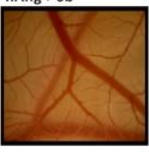

hAng $+4 \mathrm{e}$
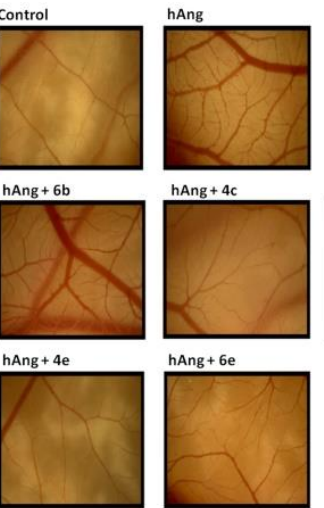

hAng $+6 e$
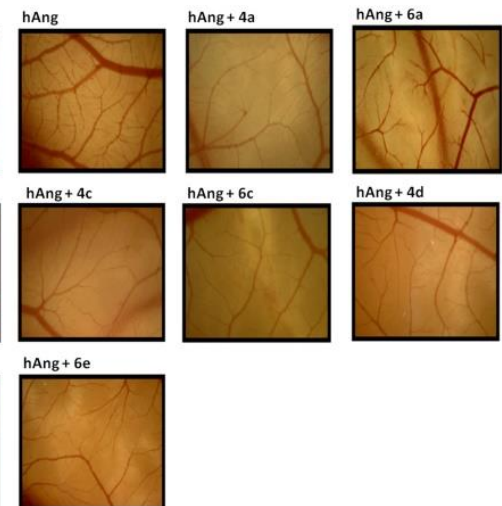

hang $+6 c$
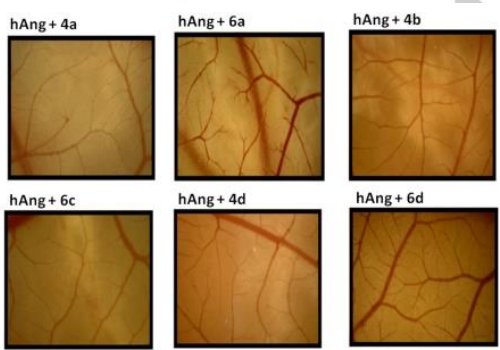

Figure 2. Inhibition of the Pyr1-hAng (2nM) angiogenic activity by $6 \mathrm{nM}$ of compounds $4 a, 4 b, 4 c, 4 d, 4 e, 6 e, 6 d, 6 c, 6 a$, and $6 b$ in the CAM assay.

We have also analyzed the effect of compounds $4 \mathbf{a}-\mathbf{e}$, and $\mathbf{6 a - e}$, on the branching of the vessels and the effect is very similar to their effect on the total length of the vessels. The diagram on the

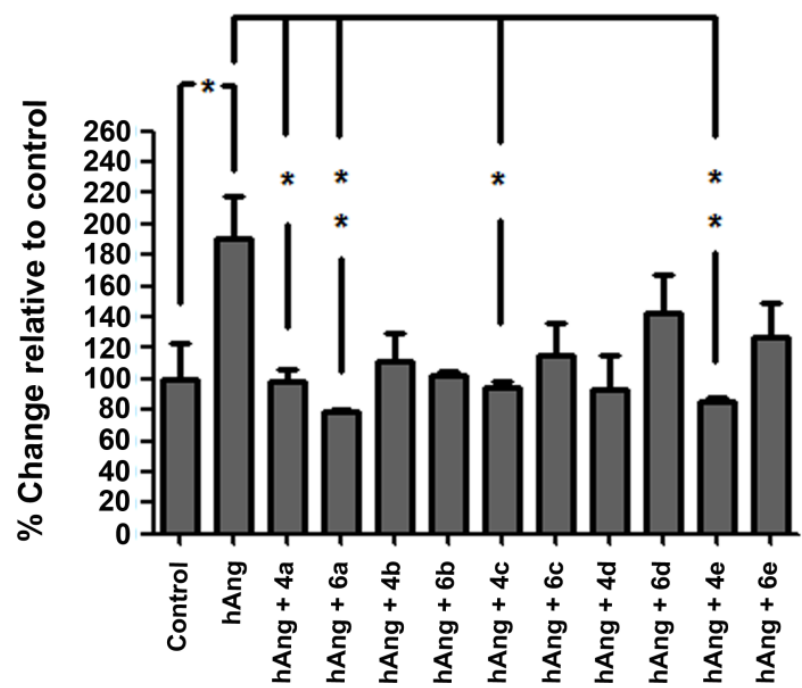

effect on branching of the vessels by compounds $4 \mathbf{a}-\mathbf{e}$, and $\mathbf{6 a - e}$, is now presented in the supporting information file (Figure S1).

Figure 3. Total length of the vessels measured using the ImagePC image analysis software (Scion Corporation, Frederick, MD).

In vitro studies

Since compounds $\mathbf{4 a}$ and $\mathbf{6 a}$ strongly inhibited the angiogenic activity of Pyr1-hAng in vivo, the next step was to check whether $4 \mathbf{a}$ and $\mathbf{6 a}$ were capable of inhibiting the ribonucleolytic activity of Pyr1-hAng in vitro. Using the precipitation assay as described in the Experimental Section, for monitoring the inhibition of the ribonucleolytic activity of Pyr1-hAng, we observed that the concentration of $\mathbf{4 a}$ and $\mathbf{6 a}$ at which $50 \%$ inhibition of ribonucleolytic activity of angiogenin occurs $\left(\mathrm{IC}_{50}\right)$ is $1 \mathrm{mM}$ and 0.7 $\mathrm{mM}$, respectively. $\mathbf{4 a}$ and $\mathbf{6 a}$ are more potent than other common Pyr1-hAng pyrimidine mononucleotide inhibitors that have been studied, 2'-CMP $\left(\mathrm{K}_{\mathrm{i}}=8.7 \mathrm{mM}\right)^{[10 \mathrm{~b}]}, 2^{\prime}-\mathrm{UMP}\left(\mathrm{K}_{\mathrm{i}}=13.1 \mathrm{mM}\right)^{[10 \mathrm{~b}]}$ and 3'CMP $\left(\mathrm{K}_{\mathrm{i}}=13.0 \mathrm{mM}\right)^{[10 \mathrm{~b}]}$. However, the measured $\mathrm{IC}_{50}$ value is rather high indicating that the disruption in the enzymatic activity might not be the reason for the significant decrease of the angiogenic activity of Pyr1-hAng observed in vivo.

NMR spectroscopy

The level of the in vitro potency of $\mathbf{4 a}$ and $\mathbf{6 a}$ does not seem to be consistent with the strong in vivo inhibitory potency against Pyr1hAng angiogenicity. Therefore, we shifted our interest towards the binding of $4 a$ and $6 a$ to hAng. NMR spectroscopy has the ability to provide structural information for the identification of binding sites even for low-affinity protein-ligand complexes ${ }^{[23]}$. Having gone through the characterization of Met(-1)-hAng through highresolution $\mathrm{NMR}^{[24]}$, we attempted to exploit the capabilities of NMR in the study of transient complexes, and studied the binding of $4 a$ and $6 a$ by measuring the ${ }^{15} \mathrm{~N},{ }^{1} \mathrm{H}-\mathrm{HSQC}$ spectra of ${ }^{15} \mathrm{~N}$ labeled Met(-1)-hAng in the presence of varying amounts of the ligand. For comparison purposes, we have additionally analyzed the binding of known hAng inhibitors, 2'-UMP[10b], 3'-CMP[10b], $\mathrm{NADPH}^{[25]}$, and $5^{\prime}-\mathrm{ATP}^{[10 \mathrm{~b}]}$ using the same methodology.

${ }^{1} \mathrm{H}$ and ${ }^{15} \mathrm{~N}$ resonance perturbations of $\operatorname{Met}(-1)$-hAng residues induced by pyrimidine nucleotides 

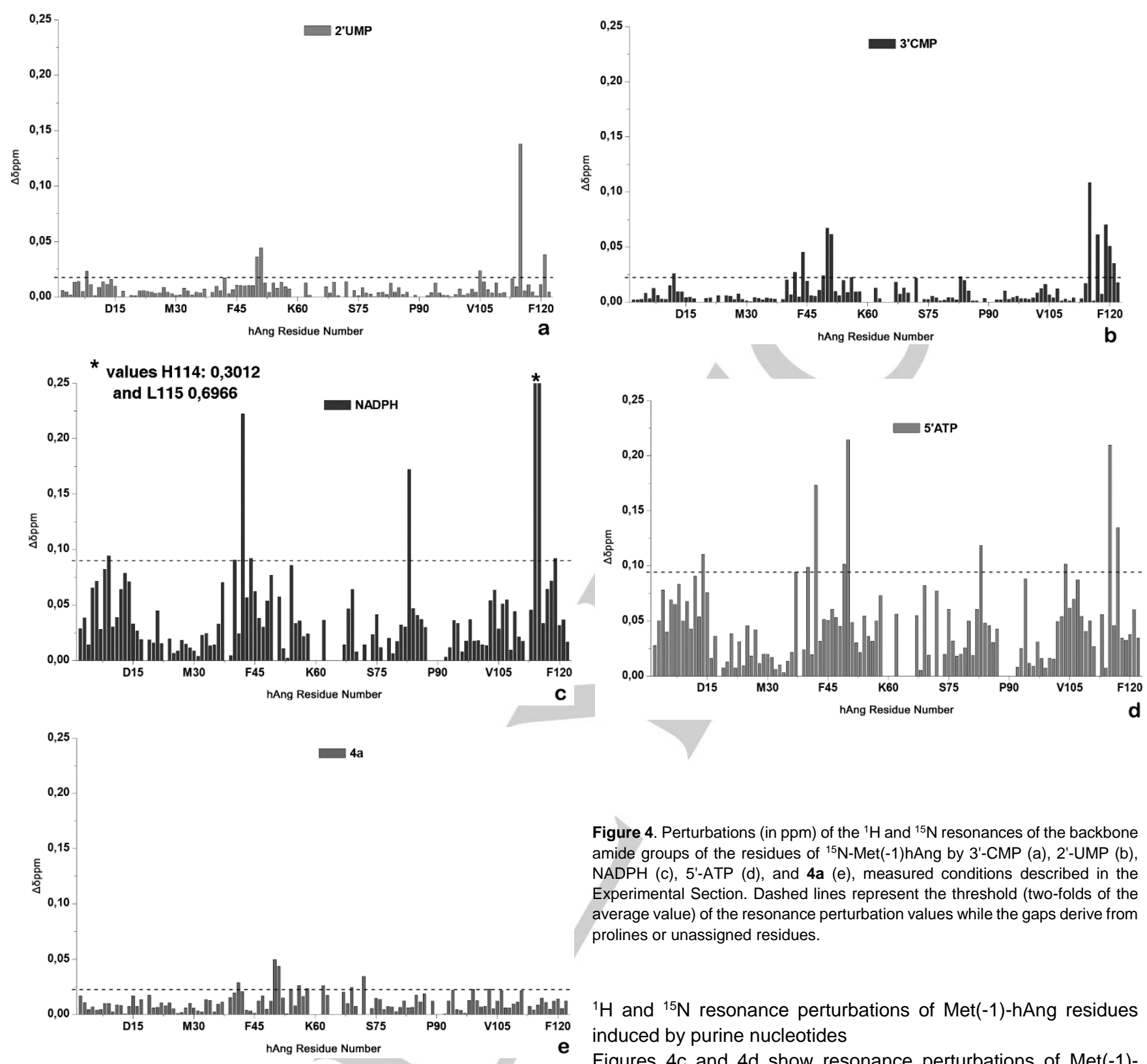

A five molar excess of $2^{\prime}-\mathrm{UMP}$ or $3^{\prime}-\mathrm{CMP}$ induced large shift perturbations of the amide protons of Gln12, Lys40, Ile42, Lys50, Arg51, lle56, Ser72, Leu115, Gln117, and Arg121 revealing a common binding pattern. Of these residues Gln12, Lys40, lle42, Leu115 and Arg121 are part of the pyrimidine binding region $\left(B_{1} R_{1} P_{1}\right.$; Figure 1$)$ of the active site of angiogenin, while $G \ln 117$ is the main obstructing residue at the pyrimidine binding subsite $\left(B_{1}\right)$. The presence of $3^{\prime}-C M P$ seems to induce larger perturbations of the backbone ${ }^{1} \mathrm{H}-{ }^{15} \mathrm{~N}$ for these residues (Figure $4 a)$ than that of 2 -UMP (Figure $4 b$ ). An additional resonance perturbation has been observed for Thr44 ( $B_{1}$ subsite) with $3^{\prime}$ CMP that has not been seen with 2'-UMP. Moreover, comparative analysis of the acquired HSQC spectra suggests that the interaction between Met(-1)-hAng and 2'-UMP or 3'-CMP falls into the fast-exchange regime on the NMR time-scale, revealing dissociation constant $\left(K_{d}\right)$ value for the two pyrimidine protein complexes circa $10^{-3}-10^{-4} \mathrm{M}$, in agreement with previously reported data ${ }^{[10 \mathrm{~b}]}$

Figure 4. Perturbations (in ppm) of the ${ }^{1} \mathrm{H}$ and ${ }^{15} \mathrm{~N}$ resonances of the backbone amide groups of the residues of ${ }^{15} \mathrm{~N}-\mathrm{Met}(-1) \mathrm{hAng}$ by $3^{\prime}-\mathrm{CMP}$ (a), 2'-UMP (b), NADPH (c), 5'-ATP (d), and 4a (e), measured conditions described in the Experimental Section. Dashed lines represent the threshold (two-folds of the average value) of the resonance perturbation values while the gaps derive from prolines or unassigned residues.

${ }^{1} \mathrm{H}$ and ${ }^{15} \mathrm{~N}$ resonance perturbations of Met(-1)-hAng residues induced by purine nucleotides

Figures $4 \mathrm{c}$ and $4 \mathrm{~d}$ show resonance perturbations of Met(-1)hAng induced by saturating amounts of NADPH (2.5 molar excess), and 5'-ATP (3 molar excess), respectively. Both molecules induced similar chemical shift changes for residues Gln12, Ile42, Arg51, Leu83, Val113, Leu115, and Gln117. Among these residues, Gln12, lle42, and Leu115 are located at the $B_{1} P_{1}$ region of the active site, while $\mathrm{G} \ln 117$ is the residue that impedes substrate binding ${ }^{[8]}$. The purine nucleotides also induced ${ }^{1} \mathrm{H}-{ }^{15} \mathrm{~N}$ resonance perturbations of His8, His13, Tyr14, Lys40, Thr44, Phe45, Asn49, Asn68, Val103, Val104, Ala106 and Cys107. His8 is part of the $P_{2}$, His 13 and Lys40 of the $P_{1}$, Thr44 of the $B_{1}$, while Asn68 is located in the $B_{2}$ subsite. Thr 7 and lle46 chemical shifts are only influenced by the presence of NADPH. In addition, NADPH induced significant resonance shifts of Arg5, Asn43, Gly48, Leu69, His114, Ser118 and Ile119 with the resonance perturbation of His 114 being the greatest (Figure 4c). Asn43 and Ser118 are at the $B_{1}$ subsite while His 114 is at $P_{1}$. Residues which exhibit significant difference at their ${ }^{15} \mathrm{~N}$ amide resonances in the presence of $5^{\prime}-A T P$, compared to those of NADPH, are Lys50, 
Glu67, and Glu108 of the $B_{2}$ subsite (Figure $4 d$ ). All the abovementioned interactions exhibit a fast exchange rate between free and bound state on the NMR time-scale, suggesting thus that these binding events are characterized by low affinity $\left(K_{d}>10^{-6} \mathrm{M}\right)$.

Purine-based inhibitors induce larger resonance perturbations of Met(-1)-hAng residues than pyrimidine-based ligands, indicating that purines exhibit higher affinity than pyrimidines in agreement with previous studies $\left.{ }^{[10 a}, 10 \mathrm{~b}, 26\right]$. Met(-1)-hAng inhibitor interactions can be assessed by comparing the pattern of ${ }^{1} \mathrm{H}$ and ${ }^{15} \mathrm{~N}$ resonance perturbations of protein residues induced upon binding. In a previous NMR study ${ }^{[27]}$ the binding of phosphate anions which occupy the catalytic $P_{1}$ subsite of angiogenin in the crystal[15] induced large perturbations of Gln12, Leu115, and Gln117. The largest perturbation induced by the binding of 5'-ATP, 2'-UMP, and 3'-CMP to Met(-1)-hAng was that for Leu115. Thus, it seems that a phosphate group of these three inhibitors occupies the catalytic $P_{1}$ subsite. This is further reinforced by the fact that the binding of $\mathbf{4 a}$ which lacks a phosphate group did not trigger any perturbation of Leu115 (see below). The binding of 5'-ATP induced a small perturbation of lle42 which is in the vicinity of $P_{1}$ indicating that the $Y$-phosphate group may interact with this residue while the $\beta$-phosphate binds at $P_{1}$. This interaction has also been observed by NMR studies of the binding of $5^{\prime}-A D P$ to angiogenin ${ }^{[27]}$. No other large perturbation of residues has been observed for the binding of $5^{\prime}$-ATP in the active site and therefore it seems that the adenosine part of the inhibitor protrudes towards the solvent. Both 2'-UMP and 3'-CMP induced large perturbation of Arg121 whereas $3^{\prime}-\mathrm{CMP}$ also triggered large perturbations of Ile119 and Phe120. These residues constitute a peripheral binding site ${ }^{[11,27-28]}$ where it seems that the pyrimidine bases are bound. 2'-UMP and 3'-CMP also induced smaller perturbations of lle42 and Thr44 (only for 3'CMP) which are part of the $B_{1}$ and we cannot rule out the possibility that the pyrimidine bases of these inhibitors bind at this subsite as well. 5'-ATP, 2'-UMP and 3'-CMP triggered perturbation of Lys 50 in a smaller scale, while $2^{\prime}$-UMP and $3^{\prime}-\mathrm{CMP}$ also induced perturbation of Arg51. These residues are outside of the active site and were found to interact with sulfate anions in the Ang-sulfate complex ${ }^{[11]}$ indicating that these three inhibitors might also have an alternative binding site. With the exception of His114, the binding of NADPH did not induce any remarkable further perturbations beyond those triggered by $5^{\prime}$-ATP. However, NADPH induced much more sizeable changes in the residue resonances than those measured with all inhibitors tested indicating much stronger binding, consistent with results from earlier kinetic studies ${ }^{[25]}$.

${ }^{1} \mathrm{H}$ and ${ }^{15} \mathrm{~N}$ resonance perturbations of Met(-1)-hAng residues induced by $\mathbf{4 a}$

The resonance perturbations induced by $4 a$ (6 molar excess, the point where no additional spectral changes were observed) are presented in Figure 4e. Remarkable differences at the resonances of the ${ }^{1} \mathrm{H}-{ }^{15} \mathrm{~N}-\mathrm{HSQC}$ peaks were observed for residues Asp41, Ile42, Lys40, Lys50, Arg51, Lys54, lle56, Glu58, Gly62, Glu67, Leu69, Ser72, Tyr94, Gly99, Val103, Ala106, and Leu111. During the incremental addition of $4 a$ there were no additional residues affected than those observed during the initial titration experiment, using the lowest concentration of $\mathbf{4 a}$ (ratio $1: 0.125)$. The NMR data of Met(-1)-hAng in the presence of $4 a$, showed low affinity binding $K_{d}>10^{-6} \mathrm{M}$, as entailed by the fast exchange rate between free and bound state on the NMR timescale. The resonance perturbations induced by $6 \mathbf{6}(10$ molar excess, Figure S2) are much weaker than those induced by $4 a$ indicating that the latter binds more tightly. Small differences at the resonances of the ${ }^{1} \mathrm{H}-{ }^{15} \mathrm{~N}-\mathrm{HSQC}$ peaks were observed for residues Gln12, Phe120 (active site), Gly62, Glu67, Asn68, Leu69, (cell-binding site), Asn49, Ile56, Cys57, Ser72, His84, Tyr94, and Val103, (Figure S2). No chemical shift perturbations of the residues that comprise the nuclear translocation site $\left.{ }_{31} R R R G L_{35}\right)$ were observed during the titration experiments with either $\mathbf{4 a}$ or $\mathbf{6 a}$. Chemical shift perturbation were also observed for the segment Ser37-lle42, with residues Ser37-Lys40 being those that exhibit the largest values (close or above the threshold, (Figure 4)). However, even though this segment is not far from the NLS (31-35), no perturbations have been observed for the NLS site.

Comparison of the ${ }^{1} \mathrm{H}-{ }^{15} \mathrm{~N}-\mathrm{HSQC}$ spectra of $4 \mathrm{a}$ with those of 2'-UMP, 3'-CMP, NADPH, and 5'-ATP reveals that the perturbations of Asp41, Gly99 and Leu111 were only induced by the presence of $4 a$ while perturbations of lle42, Lys50, Arg51 and Lys 54 were caused by the presence of all five ligands (4a, 2'-UMP, 3'-CMP, NADPH, 5'-ATP). Perturbations of residues Lys40, Ile56, Gly62, Leu69, Ser72, Tyr94, Val103 and Ala106 were also induced by the presence of 2'-UMP. With the exception of Tyr94 and Ala106 the same residues were also influenced by 3'-CMP. Changes to ${ }^{15} \mathrm{~N}-\mathrm{HSQC}$ peaks of Lys40, Leu69 and Ala106 were also observed in the presence of NADPH while the ${ }^{1} \mathrm{H}-{ }^{15} \mathrm{~N}-\mathrm{HSQC}$ peaks of Lys40, Glu58, Gly62, Glu67, Ser72, Tyr94 and Val103 were also shifted by the presence of $5^{\prime}$-ATP.

With the exception of Lys40 and Ile42, 4a did not induce any other resonance perturbations of the same active site residues of Met(-1)-hAng as 5'-ATP, NADPH, 2'-UMP and 3'-CMP. Therefore, it seems that $4 a$ does not bind only at the active site of the enzyme. The perturbations of Lys 40 and lle42 (Figure 5) are the smallest among those induced by $\mathbf{4 a}$, further supporting the existence of another binding site for this inhibitor. The most structurally significant perturbations are those of Glu58, Gly62, Glu67 and Leu69 (Figure 5). These residues are part of the cell binding site of angiogenin ${ }^{[8]}$ suggesting that this is the other binding site of $4 \mathbf{a}$. The small perturbations of Lys40 and lle42 imply that $\mathbf{4 a}$ also binds at the active site in agreement with the expression of inhibition of the ribonucleolytic activity by $\mathbf{4 a}$. 


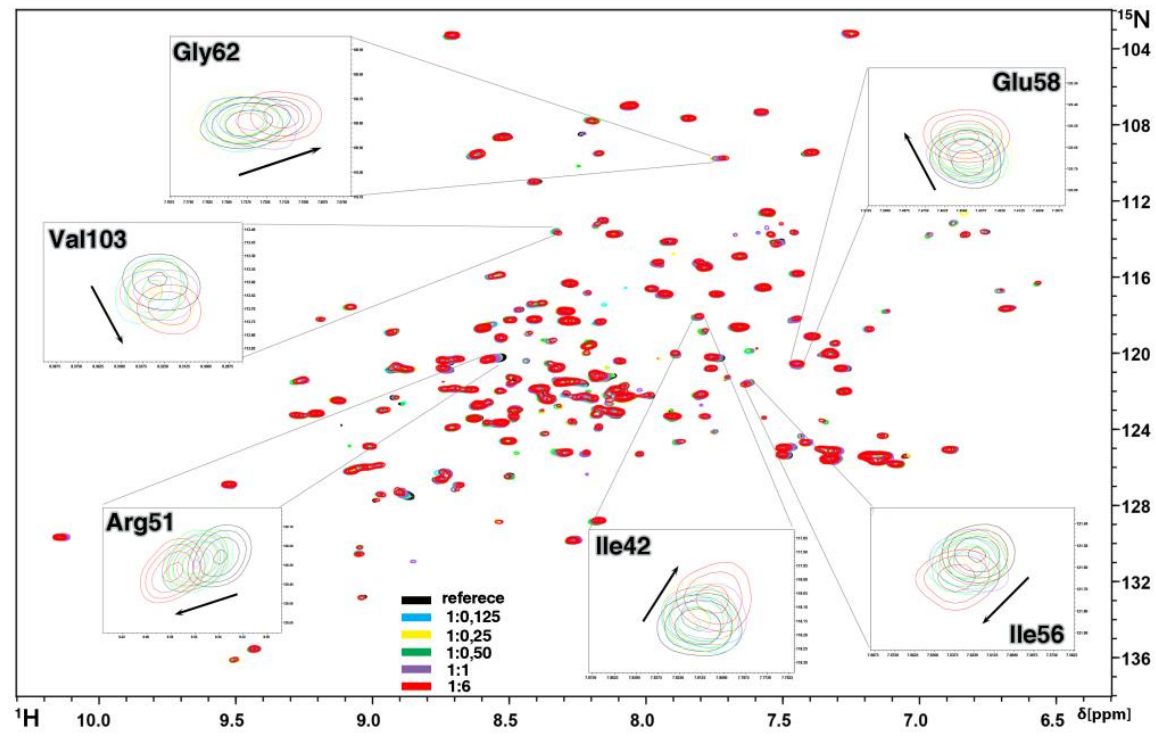

Figure 5: Titration of Met(-1)-hAng with 4a monitored by NMR spectroscopy. Superimposition of the 15N, 1H HSQC acquired spectra in absence (black) and in presence of successive additions of compound 4a (color represents each molar ratio, 1:0.125 - light blue, 1:0.25 - yellow, 1:0.5 - green, 1:1 - purple, 1:6 - red). Specific regions are magnified and correspond to polypeptide's amino acids implicated in the $4 a$ binding.
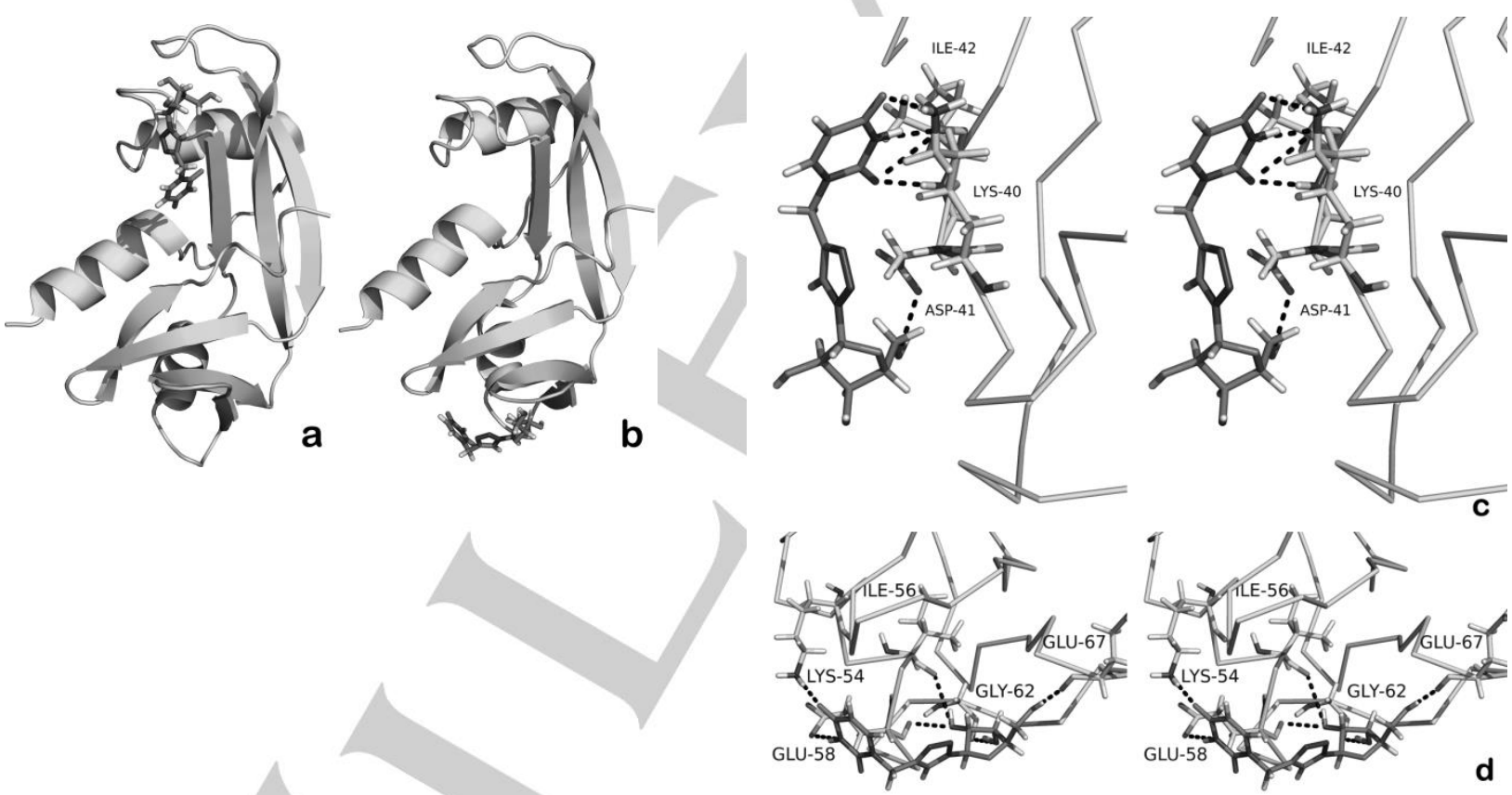

Figure 6. The binding modes of $4 a$ to hAng derived from NMR and modeling studies. (a) The structural model of the hAng-4a complex derived from the fourth ranked pose of IFD 1 calculations (Table 2) targeting the active site; (b) the structural model of the hAng-4a complex derived from the top-ranked pose of IFD-2b calculations targeting the cell binding site; (c) stereo views of the interactions of $4 a$ with hAng at the cell binding site; (d) stereo views of the interactions of $4 a$ with hAng at the active site; hAng residues are drawn as ball-and-stick models, while hydrogen bonds are indicated by dashed lines. Hydrogen bonds were assigned using Maestro ${ }^{[29]}$ default settings throughout: maximum distances $2.8 \AA$, donor and acceptor minimum angles of $120^{\circ}$ and $90^{\circ}$, respectively.

Modelling of the binding modes of $\mathbf{4 a}$ in complex with Pyr1-hAng Computational induced-fit docking analysis ${ }^{[29-30]}$ was used to derive three dimensional structures for Pyr1-hAng in complex with 4a. These calculations were performed as described in the Experimental Section targeting interactions at the active and cell binding sites. The results of the IFD calculations in terms of
GlideScore, Prime Energy and IFDscore are shown in Table 2. The fourth ranked pose from IFD 1 calculations that targeted interactions at the active site matches the experimental NMR perturbations. In this binding location of hAng (Figure 6a), 4a forms hydrogen bond interactions with the main chain atoms of Ile42 and with the side chain atoms of Lys40, and Asp41 (Figure $6 b)$ in agreement with the NMR observations. 
Using a similar standard IFD protocol for the cell binding site failed to find a docking solution compatible with the NMR data. Accordingly, more extensive conformational sampling of this binding site was explored, as described for the IFD-2a and IFD$\mathbf{2 b}$ calculations in the Experimental Section. The top-ranked pose from IFD-2b exhibited binding features in excellent agreement with NMR data. In this binding hAng location (Figure 6c), the ligand forms hydrogen bond interactions with Lys54, lle56, Glu58, Gly62, and Glu67 (Figure 6d) in the cell binding site. Each of these residues, according to the NMR data, seem to be implicated in protein-inhibitor interaction, since residues' ${ }^{1} \mathrm{H}-{ }^{15} \mathrm{~N}$ resonances are affected during the titration as described in detail above.

Table 2. Results of the induced fit docking calculations ${ }^{[a]}$

\begin{tabular}{llllll}
\hline Binding Site & $\begin{array}{l}\text { IFD } \\
\text { Protocol }\end{array}$ & $\begin{array}{l}\text { Pose } \\
\text { Rank }\end{array}$ & GlideScore & $\begin{array}{l}\text { Prime } \\
\text { Energy }\end{array}$ & IFDscore \\
\hline Active Site & IFD 1 & 1 & -5.92 & -5157.5 & -263.8 \\
& & $4^{*}$ & -4.72 & -5146.9 & -262.1 \\
Cell Binding & IFD 2a & 1 & -6.42 & -5161.5 & -264.5 \\
Site & & 17 & -4.34 & -5148.7 & -261.8 \\
& & 17 & -6.62 & -5157.2 & -264.5 \\
& IFD 2b & $1^{*}$ & & & \\
& & & & & \\
\end{tabular}

[a] Calculations as described in Experimental Section. The NMR consistent predicted protein ligand complexes are highlighted with an asterisk * in italics. Units are $\mathrm{kcal} / \mathrm{mol}$.

Using a similar standard IFD protocol for the cell binding site failed to find a docking solution compatible with the NMR data. Accordingly, more extensive conformational sampling of this binding site was explored, as described for the IFD-2a and IFD$\mathbf{2 b}$ calculations in the Experimental Section. The top-ranked pose from IFD-2b exhibited binding features in excellent agreement with NMR data. In this binding hAng location (Figure 6c), the ligand forms hydrogen bond interactions with Lys54, lle56, Glu58, Gly62, and Glu67 (Figure 6d) in the cell binding site. Each of these residues, according to the NMR data, seem to be implicated in protein-inhibitor interaction, since residues' ${ }^{1} \mathrm{H}^{-15} \mathrm{~N}$ resonances are affected during the titration as described in detail above. The flexibility of the residues in the cell-binding site meant that there were significant conformational changes in the cell binding site from the crystal structure of Pyr1-hAng used as input. Considering that we refined complexes with a common base-line energy (same protein starting conformation), it is predicted by Prime Energy that these conformational changes for the experimentally consistent cell binding site protein-ligand pose ($5157.2 \mathrm{kcal} / \mathrm{mol}$ ) result in a more favorable complex energy compared to its active site equivalent $(-5146.9 \mathrm{kcal} / \mathrm{mol})$. In a similar manner, and consistent with the proposal of the cell binding site being the other binding site of the inhibitor, the IFDscores for the experimentally consistent poses are -264.5 and $-262.1 \mathrm{kcal} / \mathrm{mol}$ for the cell-binding and active sites, respectively, with a difference of $2 \mathrm{kcal} / \mathrm{mol}$ considered significant (Schrödinger, personal communication). Towards further validating the novel binding of $\mathbf{4 a}$ at the cell-binding site, a $100 \mathrm{~ns}$ MD simulation was performed. Small and stable RMSD fluctuations of the Ca (average RMSD $=0.258 \AA$ ) and protein sidechains (average RMSD $=0.489 \AA$ ) over the simulation production run were observed as displayed in Figure 7 . Figure 8 shows the proteinligand hydrogen bond contacts as a fraction of their occurrence throughout the simulation; the major contacts with residues (Lys54, Glu67, Gly62, Glu58, lle56) are in excellent agreement with experiment, ranging from hydrogen bonding with Lys54
$94.3 \%$ of the time to $34.7 \%$ for lle 56 . Additionally, clustering of binding site conformations resulted in one dominant cluster $(99 \%$ of frames) consistent with the stability of $\mathbf{4 a}$ binding at the site.

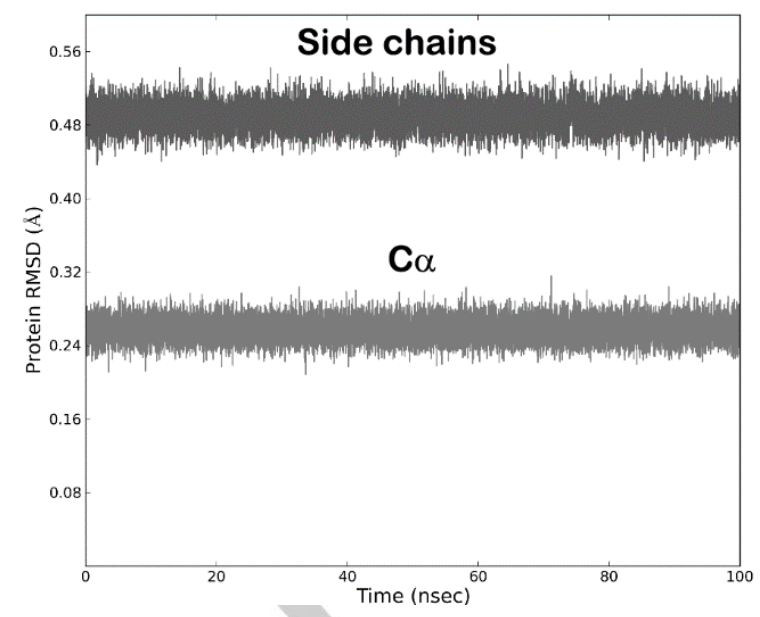

Figure 7. RMSD of protein $\mathrm{C} \alpha$ and sidechain atoms with respect to time from a 100 ns molecular dynamics simulation of $\mathbf{4 a}$ bound at the Pyr1-hAng cellbinding site. The uniform and small fluctuations around equilibrium values reflect the stability of the system over the course of the simulation.

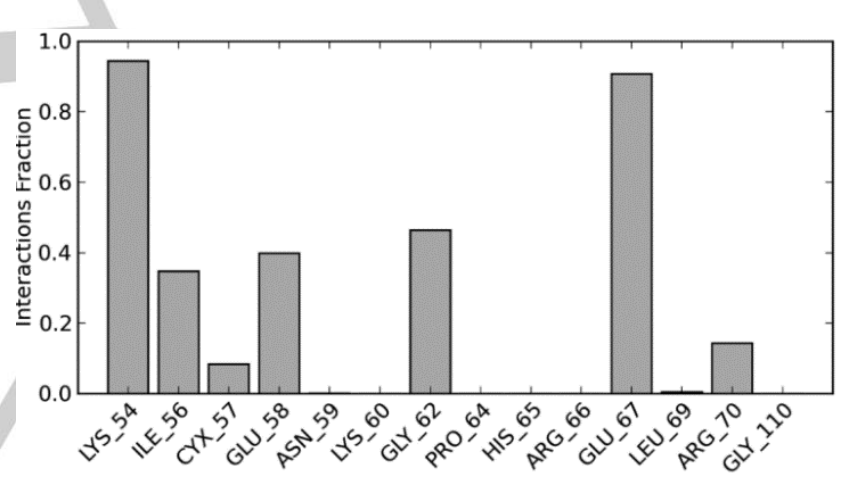

Figure 8. Hydrogen bond contacts of 4 a with amino acid residues at the Pyr1hAng cell-binding site from a 100 ns molecular dynamics simulation. Contacts are expressed as a fraction of their occurrence during the simulation.

Finally, to probe whether the binding of $\mathbf{4 a}$ at the two binding sites occurs sequentially or simultaneously, additional IFD calculations of a $\mathbf{4 a}$ ligand to the active site was performed using representative complexes from the MD simulation, retaining $\mathbf{4 a}$ bound at the cell-binding site. These docking calculations revealed a similar experimentally consistent binding pose of $\mathbf{4 a}$ (hydrogen bond interactions with Cys39, Lys40, Asp41, lle42) to that obtained in the original IFD 1 calculations (ligand heavy atom RMSD of $1.879 \AA$ ). Interactions with protein residues are in excellent agreement with the NMR data since the NMR titration experiments revealed that chemical shift perturbations of the residues of both regions (active site and cell binding site) are observed simultaneously even at the lowest ligand concentration (ratio protein:ligand $=1: 0.125$ ). This suggests the potential for binding to occur simultaneously; however, crystallographic evidence would fully confirm this to be the case. There have been numerous reports of the successful application of IFD to predict 
significant ligand dependent conformational changes in agreement with experiment together with their effects on biochemical pathways and mechanisms ${ }^{[31]}$. Likewise, here we have been able to report an additional success, highlighting the potential of the concerted use of IFD with careful parameter selection/application in combination with experimental NMR data, to elucidate new and previously unidentified binding sites on protein/enzyme surfaces. MD simulation, as was applied here, can be used to further validate newly predicted geometries.

\section{Conclusions}

In summary, we have provided evidence for the strong antiangiogenic potency in vivo of a series of ten RNase A inhibitors (ribofuranosyl pyrimidine nucleosides and their corresponding 1,2,3-triazole derivatives). The 1,2,3-triazole 5substituted uracil furanonucleosides were more potent than the $C-5$ substituted uridines. However, the strong antiangiogenic potency of these compounds was not consistent with their enzymatic inhibition in vitro. NMR data together with induced-fit docking calculations and MD simulation revealed a novel binding mode for $\mathbf{4 a}$ at $h A n g$.

Moreover, since NMR titration experiments revealed that chemical shift perturbations of the residues of both regions (active site and cell binding site) are observed simultaneously even at the lowest ligand concentration, and docking calculations are in agreement with a binding to occur simultaneously. The larger chemical shift changes observed for the residues of the cell binding site with respect to those of the active site, could possibly be indicative for stronger binding, which may be related with higher specificity

The difference in binding data between NMR and biochemical assays may be attributed to the fact that the NMR study was performed in a $\mathrm{CH}_{3} \mathrm{COONa}(50 \mathrm{mM})$ buffer with $\mathrm{pH} 5.0$, while the biochemical in vitro and in vivo assays were performed in a MES buffer $(100 \mathrm{mM})$ supplement with $100 \mathrm{mM} \mathrm{NaCl}$ at $\mathrm{pH} 6.0$ and incell, respectively, where completely different experimental conditions were applied. Therefore, the protonation states of many amino acids side chains, which are expected to be implicated in the protein - ligand interaction, are different in these three different solutions. It is well-established in NMR, that known binding events, characterized as strong with $K_{d}<10^{-9} \mathrm{M}$ are studied by NMR and $K_{d} s$ found in $\sim 10^{-3}$ to $10^{-6} \mathrm{M}$.

This is the first time that a nucleoside is reported to exert dual inhibitory action on hAng, blocking both the entrance of hAng into the cell and also its ribonucleolytic activity. The output of this work will therefore facilitate the rational design and development of new anti-angiogenic compounds targeting the angiogenin cell binding site.

hAng seems to play key roles in a plethora of biological processes and is also implicated in almost every step of tumorigenesis. It has thus become a validated diagnostic tool, prognosis and therapymonitoring marker in cancer ${ }^{[4,32]}$ for physicians ${ }^{[33]}$, as well as a validated pharmaceutical target for the development of new anticancer agents ${ }^{[34]}$.

The direct relationship between the ribonucleolytic and angiogenic activities of hAng implied that potent and selective inhibitors of its enzymatic action could be also able to inhibit its biological activity ${ }^{2}$. However, blocking only the enzymatic active site may not be efficient because it could not probably prevent the protein interactions that $\mathrm{hAng}$ forms with different protein partners which is an important regulatory mechanism for the diverse cellular functions of hAng that promote cancer establishment and progression. We have recently performed a proteomic analysis of hAng interactions by mass spectrometry (MS) ${ }^{[35]}$ and identified, among others, proliferating cell nuclear antigen (PCNA) as a novel hAng protein partner. PCNA, which forms a ring around the DNA regulating DNA replication, plays a central role in determining both tumour progression and the outcome of anticancer treatment ${ }^{[36]}$.

Inhibitors like 4a that present dual action and can block both the enzymatic activity and mainly the cell binding site, will have the advantage of blocking the biological action of hAng, regardless of the cause, since they will prevent any interaction with its target cells. Furthermore, such inhibitors, will circumvent the caveat of neutralizing all circulating hAng in the plasma where its concentration is significantly high $(\sim 250 \mathrm{ng} / \mathrm{mL})^{[34]}$, and the amount of inhibitors needed to directly target hAng may be unreachable. Finally, since normal cells do not require hAng to survive and grow and hAng's internalization and subsequent nuclear translocation occur only in cancer cells and in proliferating endothelial cells, blocking the cell binding site of hAng will selectively inhibit the growth and proliferation of cancer cells and proliferating endothelial cells but spare normal cells, presenting thus less side-effects and lower toxicity ${ }^{[34]}$.

\section{Experimental Section}

Overexpression and purification of ${ }^{15} \mathrm{~N}$ - and ${ }^{15} \mathrm{~N} /{ }^{13} \mathrm{C}$-labeled and unlabeled Met(-1)-hAng: The construction of the recombinant vector containing the gene of $h A n g$ and the purification procedure of the protein were described elsewhere[11]. Labeled Met(-1)-hAng with ${ }^{15} \mathrm{~N}$ - and ${ }^{15} \mathrm{~N} /{ }^{13} \mathrm{C}$ was prepared as previously described ${ }^{[24]}$.

Angiogenic activity:Met(-1)-hAng was converted to the authentic Pyr1 human form (Pyr1-hAng) which contains a pyroglutamic acid residue at position 1 as described previously ${ }^{[37]}$. The inhibitory potency of $4 a-4 e$ and $6 \mathrm{a}-6 \mathrm{e}^{[21]}$, on Pyr1-hAng induced angiogenesis, was assessed by using the CAM assay ${ }^{[3]}$. Briefly, Leghorn fertilised eggs were incubated for 4 days at $37^{\circ} \mathrm{C}$, when a window was opened on the egg shell, exposing the CAM. The window was covered with tape and the eggs were returned to the incubator until day 9 , when the test molecules were applied. Pyr1-hAng alone or in combination with the inhibitors were added as a solution in a final volume of $60 \mu \mathrm{L}$, at day 9 of chicken embryo development, on an area of $1 \mathrm{~cm}^{2}$ of the CAM, restricted by a plastic ring. In a different group of eggs, which was used as control, only PBS was applied. The inhibitors alone were also tested for antiangiogenic activity in the CAM assay. After $48 \mathrm{~h}$ of incubation at $37^{\circ} \mathrm{C}$, images were taken through a stereoscope equipped with a digital camera and the total length of the vessels was measured using the ImagePC image analysis software (Scion Corporation, Frederick, MD). Assays for each test sample were carried out three times and each experiment contained 6 eggs per data point.

Precipitation assay with human angiogenin: Inhibition of the ribonucleolytic activity of Pyr1-hAng was quantified following the Bond method ${ }^{[39]}$ as used in other studies ${ }^{[40]}$. Pyr1-hAng solution was determined spectrophotometrically using absorption coefficient $\varepsilon_{280 \mathrm{~nm}}=12500 \mathrm{M}^{-1} \mathrm{~cm}^{-1}$ [41]. The reaction buffer was $0.1 \mathrm{M} \mathrm{MES}, 0.1 \mathrm{M} \mathrm{NaCl}, \mathrm{pH} 6.0$, and the final volume of the reaction was $300 \mu \mathrm{L}$. The final concentrations of Pyr1-hAng, tRNA, and BSA in the reaction were $1 \mu \mathrm{M}, 10 \mu \mathrm{M}$, and $3 \mu \mathrm{M}$, respectively. The inhibitors, $4 \mathrm{a}$ or $6 \mathrm{a}$, were pre-incubated with Pyr1-hAng at $37^{\circ} \mathrm{C}$ for 30 minutes. The reaction was stopped six hours later, by the addition of $300 \mu \mathrm{L}$ of fresh ice-cold solution of $40 \mathrm{mM}$ lanthanum nitrate, $6 \%(\mathrm{v} / \mathrm{v})$ perchloric acid. Stopped reactions were maintained on ice for $15 \mathrm{~min}$, and insoluble tRNA was removed by centrifugation at $4{ }^{\circ} \mathrm{C}$ for $15 \mathrm{~min}$ at maximum speed on a bench centrifuge. The amount of cleaved tRNA was determined from the absorbance of the supernatant fraction at wavelength 
of $260 \mathrm{~nm}\left(\mathrm{~A}_{260}\right)$. A blank run (without enzyme) was also conducted and the $A_{260}$ value measured from this blank was subtracted from the corresponding reaction samples to eliminate any endogenous tRNA hydrolysis.

NMR experiments:To investigate the binding of Met(-1)-hAng with $4 \mathrm{a}$ and $6 \mathrm{a}$ and to compare it to that of other pyrimidine (2'-UMP and 3'-CMP) and purine (NADPH, 5'ATP) nucleosides, we performed titration experiments followed by the acquisition of $2 \mathrm{D}{ }^{1} \mathrm{H}-{ }^{15} \mathrm{~N}$ HSQC NMR spectra. Analysis of the resonances, which are influenced during the titration experiments allows the identification of residues that are affected by the binding of ligands to Met(-1)-hAng. We conducted five different NMR titration experiments of ${ }^{15} \mathrm{~N}$ labeled protein $(0.4-0.6 \mathrm{mM})$, one for each ligand. Measurements were performed in a buffer consisting of $50 \mathrm{mM}$ sodium acetate, $0.2 \mathrm{mM}$ EDTA, $\mathrm{pH}$ 5.0, as described in our previous study for the sequence-specific assignment of Met(-1)-hAng ${ }^{1} \mathrm{H},{ }^{15} \mathrm{~N}$ and ${ }^{13} \mathrm{C}$ resonances ${ }^{[24]}$. This buffer does not affect the enzymatic activity of angiogenin, as reported in previous NMR studies ${ }^{[27]} .{ }^{1} \mathrm{H}-{ }^{15} \mathrm{~N}$ HSQC spectra were recorded after each addition of the nucleosides at $298 \mathrm{~K}$ on a Bruker Avance III High-Definition, four-channel $700 \mathrm{MHz}$ NMR spectrometer equipped with a cryogenically cooled $5 \mathrm{~mm}{ }^{1} \mathrm{H} /{ }^{13} \mathrm{C} /{ }^{15} \mathrm{~N} / \mathrm{D}$ Z-gradient probe. The incremental addition of each putative ligand was completed at the point (concentration) that no spectral changes were observed. Combined chemical shift perturbations were calculated using the equation: $\Delta \mathrm{av}=$ $\left[\left(\Delta \delta_{H N}\right)^{2}+\left(\Delta \delta_{N} / 5\right)^{2}\right]^{1 / 2}[23]$.

Induced-fit Docking analysis of Pyr1-hAng - 4a complex: The 4a ligand was prepared for calculations using LigPrep $3.6^{[29]}$ with the OPLS3 forcefield ${ }^{[42]}$, while hAng was prepared using the native Pyr1-hAng crystal structure (PDB id: 5EOP ${ }^{[11]}$ ). The initial setup of the Pyr1-hAng structure was performed using Schrodinger's "Protein Preparation Wizard" [29]. Water molecules were deleted, bond orders assigned, and hydrogen atoms added, with protonation states for basic and acidic residues based on residue PROPKA $p K_{a}$ values at normal $\mathrm{pH}$ (7.0). Subsequent optimization of hydroxyl groups, histidine protonation states and $\mathrm{C} / \mathrm{N}$ atom "flips", and side-chain O/N atom flips of Asn and Gln was based on optimizing hydrogen bonding patterns. Finally, an "Impref" minimization using the OPLS3 force field ${ }^{[42]}$ was performed to remove steric clashes and bad contacts but with heavy atoms constrained to within $0.3 \AA$ (RMSD) of their crystallographic positions. Schrödinger IFD calculations ${ }^{[30]}$ of $\mathbf{4 a}$ to hAng were performed to predict protein-ligand binding interactions consistent with the observed NMR residue perturbations. Accordingly, two hAng binding regions were targeted. The first IFD calculation (IFD 1 Active Site) aimed at predicting the binding close to the active site; the centroid of residues Lys40, Asp41, Ile42 and Tyr94 which exhibited NMR perturbations in this region were used to define the box centre. The IFD itself consisted of three calculation Stages (I-III). In Stage I, docking was performed using Glide 7.1 standard-precision (SP) docking with default parameters and with a maximum of 20 ligand binding poses saved. In Stage II, residues within $5 \AA$ of the initial protein-ligand poses were refined using Prime 4.4. Finally, in Stage III up to 20 protein structures within 30 $\mathrm{kcal} / \mathrm{mol}$ of the lowest-energy complex structure were used for Glide-SP ligand re-docking calculations. The second IFD calculation (IFD $2 \mathrm{a}-$ Cell Binding Site) aimed at predicting the binding close to the cell binding site and required a more extensive approach to sampling the receptor-ligand conformational space to identify the binding interactions consistent with the NMR data. The centroid of residues Glu58 and Glu67 at opposite ends of the cell binding site were used to define the box centre. IFD parameters were applied as for the active site region except for the following: in Stage I residues 59-70 were mutated to Ala for the initial Glide-SP docking; in Stage II residues were rebuild and refined using Prime within $7 \AA$ of these initial protein-ligand poses for the final (Stage III) redocking calculations. One receptor-ligand pose from this protocol gave a pose partly consistent with the experimental NMR data which involved hydrogen bond contacts from ligand to Glu67 $\mathrm{O}$ and the pyrimidine $\mathrm{CO}$ with the Lys54 sidechain $\mathrm{NH}_{3}{ }^{+}$. Using this pose (centroid of $4 \mathrm{a}$ in binding site used to define the grid box centre) and these hydrogen bonds as constraints for a second IFD calculation (IFD $2 b$ - Cell Binding Site), the IFD was repeated without mutations and the same setting as for the IFD 1 calculation. The resulting receptor-ligand poses from the IFD calculations were analysed in terms of structure and agreement with experiment (NMR chemical shift perturbation), ligand re-docking GlideScore (GS), and IFDscore (ligand redocking GS + 5\% Prime energy from the refinement calculation).

Molecular Dynamics Simulation of Pyr1-hAng - 4a cell-binding complex: The experimentally consistent IFD $2 \mathrm{~b}$ predicted binding pose of 4a at the cell-binding site was used as input for MD using Desmond version 4.6. The initial set-up involved soaking the system with a pre-equilibrated TIP3P model orthorhombic water box (sides $a=66.66 \AA$, $b=55.16 \AA$, $c=$ $50.30 \AA$, volume $184,951 \AA^{3}$ ) allowing for a $10 \AA$ buffer region between protein and box sides, and neutralizing the system with $\mathrm{Cl}^{-}$ions. This setup procedure produced 5130 water molecules with the final system consisting of 17,368 atoms. Forcefield parameters for the protein-ligand systems were assigned using the OPLS3 forcefield ${ }^{[42]}$. Heavy atom bond lengths with hydrogens and the internal geometry of water molecules were constrained using the SHAKE algorithm. Periodic boundary conditions (PBC) were applied and a cut-off of $9.0 \AA$ for non-bond interactions, with electrostatic interactions treated using the Particle Mesh Ewald (PME) method $^{[43]}$. Prior to the MD production run, a relaxation protocol was employed as follows: (a) 2000 steps minimization with the solute restrained and a loose convergence criteria of $50 \mathrm{kcal} \mathrm{mol}^{-1} \AA^{-1}$; (b) 2000 steps minimization (no restraints) with a convergence criteria of $1 \mathrm{kcal} \mathrm{mol}^{-1} \AA^{-1}$ (c) a short Brownian dynamics 50 -ps simulation using a temperature $(T)$ of $10 \mathrm{~K}$ with solute heavy atoms restrained; (d) a 50-ps simulation in the NVT ensemble using $T=10 \mathrm{~K}$ (thermostat relaxation constant $=0.1 \mathrm{ps}$ ) with solute heavy atoms restrained (e) a 100-ps simulation in the NPT ensemble using $\mathrm{T}=10 \mathrm{~K}$ (thermostat relaxation constant $=0.1 \mathrm{ps}$ ) and pressure $(P)=1 \mathrm{~atm}$ (barostat relaxation constant $=50 \mathrm{ps}$ ) with solute heavy atoms restrained; (e) solvate pocket as necessary (f) a 100-ps simulation in the NPT ensemble $(T=300 \mathrm{~K}$; thermostat relaxation constant $0.1 \mathrm{ps} ; \mathrm{P}=1 \mathrm{~atm}$; barostat relaxation constant $50.0 \mathrm{ps}$ ) with solute heavy atoms restrained; and $(\mathrm{g})$ a 200-ps simulation in the NPT ensemble ( $\mathrm{T}=$ $300 \mathrm{~K}$; thermostat relaxation constant $0.1 \mathrm{ps} ; \mathrm{P}=1 \mathrm{~atm}$; barostat relaxation constant 2.0 ps) with no restraints. For all the above, the relaxation constants relate to Berendsen thermostats and barostats. Following the relaxation, a 100 ns production run in the NPT ensemble $(T=300 \mathrm{~K}$, thermostat relaxation time $=1.0 \mathrm{ps} ; \mathrm{P}=1 \mathrm{~atm}$; barostat relaxation time $=$ 2.0 ps) was performed using a Nose-Hoover thermostat and MartynaTobias-Klein barostat ${ }^{[44]}$. A multiple time step RESPA integration algorithm was used with time steps of 2, 2, and 6 fs for bonded, "near" non bonded, and "far" non bonded interactions, respectively. Energy and trajectory atomic coordinate data were collected every 1.2 and $5.0 \mathrm{ps}$, respectively. Visualization and analysis of the MD trajectory was performed employing Desmond Maestro's simulation analysis tools. Exploiting Maestro's Desmond trajectory clustering tool and the hierarchical cluster linkage method, clustering of 1000 trajectory frames considering the binding site conformations (ligand and protein residues within $7 \AA$ ) into 10 clusters based on atomic RMSDs was performed ${ }^{[45]}$. Using the MD representative complexes from the two largest clusters (water and $\mathrm{Cl}^{-}$ions deleted) and with $4 \mathrm{a}$ retained bound at the cell binding site, IFD was repeated for a $4 \mathrm{a}$ molecule binding to the active site using the same settings as outlined above for IFD 1 - Active Site; this allowed us to consider whether the binding of $\mathbf{4 a}$ to the active and cell-binding sites can occur simultaneously.

\section{Acknowledgements}

The assistance of Dr M. Terzenidou in the preparation of Figure 3 is greatly acknowledged. This work was supported by the Hellenic General Secretariat for Research and Technology (GSRT), through a Joint Research and Technology project between Greece and France (2010-2012) and in part by the Postgraduate Programmes "Biotechnology-Quality assessment in Nutrition and the Environment", "Application of Molecular Biology-Molecular Genetics-Molecular Markers", Department of Biochemistry and Biotechnology, University of Thessaly. Additionally, "SEE-DRUG" 
Grant (EU FP7 REGPOT CT-2011-285950

www.seedrug.upatras.gr) is acknowledged for the purchase of the $700 \mathrm{MHz}$ NMR instrument.

Keywords: Angiogenin • RNase superfamily • angiogenesis, inhibition $\bullet$ triazole nucleosides $\cdot \mathrm{NMR}$ spectroscopy $\bullet$ induced fit modelling

\section{References:}

a)J. W. Fett, D. J. Strydom, R. R. Lobb, E. M. Alderman, J. L. Bethune, J. F. Riordan, B. L. Vallee, Biochemistry 1985, 24, 5480-5486; b)S. Weremowicz, E. A. Fox, C. C. Morton, B. L. Vallee, Am. J. Hum. Genet. 1990, 47, 973981.

[2] D. J. Strydom, J. W. Fett, R. R. Lobb, E. M. Alderman, J. L. Bethune, J. F. Riordan, B. L. Vallee, Biochemistry 1985 , 24, 5486-5494.

[3] J. W. Fett, D. J. Strydom, R. R. Lobb, E. M. Alderman, J. L. Bethune, J. F. Riordan, B. L. Vallee, Biochemistry 1985 , 24, 5480-5486.

[4] J. Sheng, Z. Xu, Acta Biochim Biophys Sin (Shanghai) 2016, 48, 399-410.

[5] J. Ang, J. Sheng, K. Lai, S. Wei, X. Gao, PLoS One 2013, 8, e71487.

[6] M. M. Emara, P. Ivanov, T. Hickman, N. Dawra, S. Tisdale, N. Kedersha, G. F. Hu, P. Anderson, J Biol Chem 2010, 285, 10959-10968.

[7] K. Kishimoto, S. Liu, T. Tsuji, K. A. Olson, G. F. Hu, Oncogene 2005, 24, 445-456.

[8] D. D. Leonidas, R. Shapiro, S. C. Allen, G. V. Subbarao, K. Veluraja, K. R. Acharya, J. Mol. Biol. 1999, 285, 1209 1233.

[9] R. Shapiro, E. A. Fox, J. F. Riordan, Biochemistry 1989, 28, 1726-1732.

[10] a)A. Russo, K. R. Acharya, R. Shapiro, Methods Enymol. 2001, 341, 629-648; b)N. Russo, K. R. Acharya, B. L. Vallee, R. Shapiro, Proc. Natl. Acad. Sci. U.S.A. 1996, 93, 804-808; c)R. Y. Kao, J. L. Jenkins, K. A. Olson, M. E. Key, J. W. Fett, R. Shapiro, Proc. Natl. Acad. Sci. U. S. A. 2002, 99, 10066-10071.

[11] D. S. Chatzileontiadou, V. G. Tsirkone, K. Dossi, A. G. Kassouni, P. G. Liggri, A. L. Kantsadi, G. A. Stravodimos, N. A. Balatsos, V. T. Skamnaki, D. D. Leonidas, FEBS Lett. 2016, 590, 3005-3018.

[12] E. Boix, M. V. Nogues, C. H. Schein, S. A. Benner, C. M. Cuchillo, J. Biol. Chem. 1994, 269, 2529-2534. C. L Biochem. 1997, 250, 712-726. a)D. D. Leonidas, R. Shapiro, G. V. Subbarao, A. Russo, K. R. Acharya, Biochemistry 2002, 41, 2552-2562; b)N. Thiyagarajan, R. Ferguson, V. Subramanian, K. R. Acharya, Nat. Commun. 2012, 3, 1121.

[15] D. D. Leonidas, G. B. Chavali, A. M. Jardine, S. Li, R. Shapiro, K. R. Acharya, Protein Sci. 2001, 10, 1669-1676. a)K. A. Olson, J. W. Fett, T. C. French, M. E. Key, B. L. Vallee, Proc. Natl. Acad. Sci. U. S. A. 1995, 92, 442-446 b)K. A. Olson, T. C. French, B. L. Vallee, J. W. Fett, Cancer Res. 1994, 54, 4576-4579; c)R. Piccoli, K. A Olson, B. L. Vallee, J. W. Fett, Proc. Natl. Acad. Sci. U.S.A. 1998, 95, 4579-4583; d)K. A. Olson, H. R. Byers, M. E. Key, J. W. Fett, Int. J. Cancer 2002, 98, 923-929.

[18] a)Y. S. Gho, C. B. Chae, J. Biol. Chem. 1997, 272, 2429424299; b)Y. S. Gho, W. H. Yoon, C. B. Chae, J. Biol. Chem. 2002, 277, 9690-9694.

[19] a)J. L. Jenkins, R. Y. Kao, R. Shapiro, Proteins 2003, 50 81-93; b)J. L. Jenkins, R. Shapiro, Biochemistry 2003, 43, 6674-6687.

[20] R. T. Raines, Chem. Rev. 1998, 98, 1045-1065
V. Parmenopoulou, D. S. M. Chatzileontiadou, S. Manta, S. Bougiatioti, P. Maragozidis, D. N. Gkaragkouni, E. Kaffesaki, A. L. Kantsadi, V. T. Skamnaki, S. E. Zographos, P. Zounpoulakis, N. A. A. Balatsos, D. Komiotis, D. D. Leonidas, Biorg. Med. Chem. 2012, 20, 7184-7193.

[22] a)P. Nowak-Sliwinska, T. Segura, M. L. Iruela-Arispe, Angiogenesis 2014, 17, 779-804; b)G.-F. Hu, Proc. Natl. Acad. Sci. USA 1998, 95, 9791-9795.

[23] J. J. Ziarek, F. C. Peterson, B. L. Lytle, B. F. Volkman, Methods Enzymol. 2011, 493, 241-275.

[24] A. C. Tsika, D. S. Chatzileontiadou, D. D. Leonidas, G. A. Spyroulias, Biomol. NMR Assign. 2016, 10, 379-383. K. Kumar, J. L. Jenkins, A. M. Jardine, R. Shapiro, Biochem. Biophys. Res. Commun. 2003, 300, 81-86.

[26] N. Russo, R. Shapiro, J. Biol. Chem. 1999, 274, 1490214908.

[27] K. Tonan, P. Xu, J. L. Jenkins, A. Russo, R. Shapiro, F. $\mathrm{Ni}$, Biochemistry 2003, 42, 11137-11149.

[28] N. Russo, V. Nobile, A. DiDonato, J. F. Riordan, B. L. Vallee, Proc. Natl. Acad. Sci. U.S.A. 1996, 93, 3243-3247.

[29] L. L. C. Schrodinger, New York, 2015

[30] W. Sherman, T. Day, M. P. Jacobson, R. A. Friesner, R. Farid, J. Med. Chem. 2006, 49, 534-553.

[31] a)M. Azam, J. T. Powers, W. Einhorn, W. S. Huang, W. C. Shakespeare, X. Zhu, D. Dalgarno, T. Clackson, T. K. Sawyer, G. Q. Daley, Chem. Biol. Drug Des. 2010, 75, 223-227; b)C. E. Drakou, A. Malekkou, J. M. Hayes, C. W. Lederer, D. D. Leonidas, N. G. Oikonomakos, A. I. Lamond, N. Santama, S. E. Zographos, Proteins 2012, 80 206-220; c)D. Georgatza, V. A. Gorgogietas, P. Kylindri, M. C. Charalambous, K. K. Papadopoulou, J. M. Hayes, A.-M. G. Psarra, Int. J. Biochem. Cell Biol. 2016, 79, 277287; d)G. Wu, S. C. Vashishtha, J. C. Erve, Chem. Res. Toxicol. 2010, 23, 1393-1404.

[32] A. Tello-Montoliu, J. V. Patel, G. Y. Lip, J. Thromb. Haemost. 2006, 4, 1864-1874.

[33] a)J. Rykala, K. Przybylowska, I. Majsterek, G. PaszWalczak, A. Sygut, A. Dziki, J. Kruk-Jeromin, Pathol. Oncol. Res. 2011, 17, 809-817; b)S. K. Ramcharan, G. Y. Lip, P. S. Stonelake, A. D. Blann, Eur. J. Clin. Invest. 2013, 43, 801-808; c)C. Barcena, M. Stefanovic, A. Tutusaus, G. A. Martinez-Nieto, L. Martinez, C. GarciaRuiz, A. de Mingo, J. Caballeria, J. C. Fernandez-Checa, M. Mari, A. Morales, Sci. Rep. 2015, 5, 7916; d)A. Rozman, M. Silar, M. Kosnik, Radiol Oncol 2012, 46, 354359.

[34] S. Li, G. F. Hu, Int. J. Biochem. Mol. Biol. 2010, 1, 26-35

[35] D. S. M. Chatzileontiadou, M. Samiotaki, A. N. Alexopoulou, M. Cotsiki, G. Panayotou, M. Stamatiadi, N. A. A. Balatsos, D. D. Leonidas, M. Kontou, J. Proteome Res. 2017, 16, 3606-3622.

[36] I. Stoimenov, T. Helleday, Biochem. Soc. Trans. 2009, 37, 605-613.

[37] R. Shapiro, J. W. Harper, E. A. Fox, H. W. Jansen, F. Hein, E. Uhlmann, Anal. Biochem. 1988, 175, 450-461. C. Hadjimichael, D. Kardamakis, S. Papaioannou, Anticancer Res. 2005, 25, 1059-1065.

[39] M. D. Bond, Anal. Biochemistry 1988, 173, 166-173.

[40] a)K. S. Ghosh, J. Debnath, P. Dutta, B. K. Sahoo, S. Dasgupta, Bioorg. Med. Chem. 2008, 16, 2819-2828; b)T. K. Maiti, D. Soumya, S. Dasgupta, T. Pathak, Bioorg. Med. Chem. 2006, 14, 1221-1228; c)J. Debnath, S. Dasgupta, T. Pathak, Chemistry (Easton) 2012, 18, 1618-1627.

[41] B. Crabtree, N. Thiyagarajan, S. H. Prior, P. Wilson, S. lyer, T. Ferns, R. Shapiro, K. Brew, V. Subramanian, K. R. Acharya, Biochemistry 2007, 46, 11810-11818.

[42] E. Harder, W. Damm, J. Maple, C. Wu, M. Reboul, J. Y. Xiang, L. Wang, D. Lupyan, M. K. Dahlgren, J. L. Knight, J. W. Kaus, D. S. Cerutti, G. Krilov, W. L. Jorgensen, R. Abel, R. A. Friesner, J. Chem. Theory Comput. 2016, 12, 281-296.

[43] U. Essmann, L. Perera, M. L. Berkowitz, T. Darden, H. Lee, L. G. Pedersen, J. Chem. Phys. 1995, 103, 8577 8593. 
[44] a)G. J. Martyna, M. L. Klein, M. Tuckerman, J. Chem.

Phys. 1992, 97, 2635-2643; b)G. J. Martyna, D. J. Tobias,

M. L. Klein, J. Chem. Phys. 1994, 101, 4177-4189.

[45] J. M. Hayes, V. T. Skamnaki, G. Archontis, C. Lamprakis, J. Sarrou, N. Bischler, A. L. Skaltsounis, S. E. Zographos, N. G. Oikonomakos, Proteins 2011, 79, 703-719. 


\section{Entry for the Table of Contents}

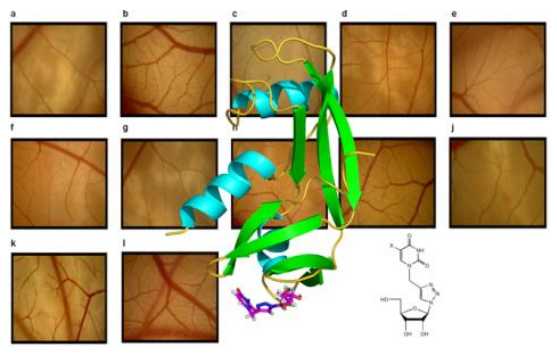

A nucleoside is reported to disrupt the biological function of human angiogenin in vivo and its enzymatic activity in vitro. NMR spectroscopy revealed simultaneous binding at the cellular and active site of angiogenin while computational modeling in line with NMR data suggested the protein-ligand interactions. 